\title{
Ironie als Instrument der „Wahrheitsfindung“
}

\section{Stephan Wolff und Hermann Müller}

Universität Hildesheim, Institut für Sozialpädagogik, Marienburger Platz 22, D-31141 Hildesheim

Zusammenfassung: In Strafverfahren finden sich auffällig viele ironische Äußerungen. In einer konversationsanalytischen Rekonstruktion dieser Passagen werden die für Ironie typische Beteiligtenstruktur und Sequentialität herausgearbeitet. Dies wiederum eröffnet Perspektiven einerseits auf ein genuin soziologisches Verständnis der Ironie, zum anderen auf die besonderen Funktionen von ironischen Äußerungen im spezifischen Verfahrenskontext. Ironie dient danach als Instrument für Verständigung und Ausgrenzung gleichermaßen; sie erleichtert die Ausbildung eines für das betreffende Verfahren relevanten kollektiven Gedächtnisses und hilft überdies die Ernsthaftigkeit des Verfahrens zu sichern.

„Mit der Ironie ist durchaus nicht zu scherzen“

(Friedrich Schlegel)

\section{Ernsthaftigkeit und Verfahren}

Obwohl das Setting wie die Abläufe bei Gericht bisweilen an Theateraufführungen erinnern, und obwohl gerade der Strafprozeß und seine Beteiligten beliebte Zielscheiben für die gesellschaftliche Witzproduktion abgeben, sind solche Verfahren im großen und ganzen eine recht ernste Angelegenheit. Ein Grund dafür liegt in den hohen Anforderungen an die Konsistenz der Darstellung bei allen Beteiligten. Niklas Luhmann (1969) bringt dies mit der verfahrenstypischen Ausrichtung auf Entscheidung in Verbindung. Im Hinblick darauf müsse sichergestellt werden, daß an die beabsichtigten und die unbeabsichtigten Informationen, welche die Beteiligten im Verlaufe der Verhandlung geben, zuverlässig angeknüpft werden kann.

\begin{abstract}
„Alle Beteiligten werden durch den Sinn und das Zeremoniell des Verfahrens angehalten, ihr Verhalten ernst zu nehmen und es als bindend zu betrachten wie eine Kette von Versprechungen, und sie tun gut, diesen Erwartungen durch Vorprüfung ihres Verhaltens Rechnung zu tragen." (Luhmann 1969: 92)
\end{abstract}

Von den professionell damit Befaßten, aber auch von den beteiligten „Laienspielern“ (also den Angeklagten und den Zeugen), erwartet man also ein gewisses Maß von Unpersönlichkeit. Alle Akteure haben sich so zu verhalten, daß ihre sonstigen sozialen Identitäten (in anderen gesellschaftlichen Funktions- bzw. Interaktions- und Organisationssystemen) nicht auf ihr Verhalten im Verfahren abfärben bzw. entsprechende Rückschlüsse erlauben. Verfahren sind darauf angelegt, „Persönlichkeit" einzufangen und zu binden. Dies geschieht u.a. da- durch, daß bestimmte, im alltäglichen Verkehr verfügbare Instrumente für die Gestaltung sozialer Situationen nur eingeschränkt verfügbar sind.

„Obgleich man Ansätze zur und Bemühungen um Distanzierung von der eigenen Rolle bei Prozeßparteien und vor allem natürlich bei Angeklagten im StrafprozeB immer wieder beobachten kann, werden ihnen durch das Zeremoniell und die offizielle Ernsthaftigkeit des Geschehens die wichtigsten Ausdrucksmittel wie Scherz, Übertreibung, Lässigkeit usw. genommen.“ (Luhmann 1969: 98f.)

Diese Feststellung ist sicherlich ein wenig zu apodiktisch formuliert. Gleichwohl trifft es auch nach unseren Beobachtungen zu, daß sich im Strafverfahren humorige Äußerungen, wie Scherze, Witze und Frotzeln ziemlich selten finden. Wenn sie überhaupt auftauchen, versehen sie die Beteiligten in aller Regel mit besonderen Markierungen (z. B.: „... wenn Sie mir die Bemerkung gestatten“). Eine bemerkenswerte Ausnahme stellen Ironisierungen dar. Ironische Bemerkungen betreffen fast ausschließlich die Aussagen bzw. Eigenschaften von Angeklagten und - bestimmten - Zeugen. Ihre primäre Zielscheibe scheint der Wahrheitsgehalt von Aussagen und damit verbunden, die Glaubwürdigkeit ihrer Urheber zu sein. Dies war der Grund, warum unsere Forschungsgruppe, die sich mit der interaktiven Konstruktion von Glaubwürdigkeit bzw. von Unglaubwürdigkeit in Strafverfahren befaßt, mit dem Phänomen der Ironie in Berührung gekommen ist.

\footnotetext{
${ }^{1}$ Unsere Ausführungen basieren auf einem Material von 36 aufgezeichneten und weiteren 20 beobachteten Strafverfahren. Die im Folgenden vorgestellten und diskutierten Beispiele für Ironie stammen aus nach konversationsanalytischer Konvention transkribierten Zeugenaussagen und aus transkribierten 'Einlassungen der Angeklagten'. Zum Zwecke der besseren Lesbarkeit haben wir die
} 
Blickt man in die einschlägige Literatur, so wird dort als für die Ironie typisch die Dialektik von augenfälliger Verdecktheit und scheinhafter Offenheit bezeichnet. Ironie, so eine typische LexikonDefinition, sei ,hinter Ernst versteckter Spott, mit dem man das Gegenteil von dem ausdrückt, was man meint, seine wirkliche Meinung aber durchblicken läßt. " Charakteristisch für die Ironie ist danach die „Schwebe zwischen Ernstnehmen und Nicht-Ernstnehmen“. Könnte man die Unangemessenheit von äußerer Form und gemeintem Sinn sofort durchschauen, würde dies die ironische Wirkung gefährden.

,Würde der Betreffende die Ironie sofort und vollständig durchschauen, so hätte sie ihren Sinn verloren, sie wäre aufgehoben und zu einer abgeblaßten, vielleicht etwas nachlässig gebrauchten Redeweise geworden. Die in der Ironie eigentlich beabsichtigte Wirkung ist vielmehr die, wo der Betroffene sie weder schlechthin durchschaut noch schlechthin auf sie hereinfällt, sondern stutzig wird - möglichst erst nachträglich, nachdem er sie zuvor für bare Münze genommen hat - und dann im Zweifel zwischen den beiden Möglichkeiten hin- und hergeworfen wird. Erst hier kommt ein für die Ironie entscheidender Zug ans Licht: daß man nicht weiß, wie weit man das ironische Verhalten ernst nehmen soll und wie weit nicht und so gerade durch diese Unsicherheit in der Schwebe gehalten wird." (Bollnow 1958: $152 \mathrm{f}$.)

Übereinstimmung herrscht in der Literatur darüber, daß der Ironisierende mit seiner Äußerung etwas feststellt, was er eigentlich so nicht meint. Ein wesentlicher Aspekt der Ironie scheint mithin darin zu bestehen, daß die ironisierende Person in ihrer Aussage neben dem offiziellen Text noch eine $\mathrm{Ge}$ genposition nicht nur mitschwingen läßt, sondern diese sogar zur wesentlichen Botschaft macht. Die Rezipienten ihrerseits müssen in die Lage versetzt werden, zwei sich widersprechende Botschaften wahrzunehmen, um die Äußerung als eine ironische erkennen zu können, und darüber hinaus die ,wirkliche Bedeutung “ des Gesagten herausfinden. ${ }^{2}$ Dies

Transkriptausschnitte vereinfacht. Alle Angaben sind anonymisiert. Wolff (1994) informiert über Perspektive und Anlage dieses Forschungsprojektes: Wolff/Müller (1995) und Wolff et al. (1996) über die wichtigsten Ergebnisse.

${ }^{2} \mathrm{Zu}$ diesen Leistungen müssen Ironisierer wie ihre Rezipienten natürlich auch von ihrer kommunikativen Kompetenz her in der Lage sein. Neben kognitiven Voraussetzungen (Kinder haben bekanntlich große Schwierigkeiten mit der Ironie!) ist damit ein gewisses Niveau sozialer Reflexivität im Interaktionssystem angesprochen. Die Ironie kann anscheinend nur in einer bestimmten kommunikativen Kultur gedeihen und goutiert werden. In Hinblick auf das historische Entstehen derartiger sozialer Reflexivität finden sich instruktive Ausführungen bei Luhmann (1980). ist nicht einfach: ironische Bemerkungen gehen bekanntlich leicht ,daneben“. Andererseits sind sich die betreffenden Autoren einig, daß die Signale, die den Rezipienten darauf hinweisen, daß die Äußerung nicht wörtlich, sondern anders zu verstehen ist, immer einigermaßen vage und unsicher bleiben müssen. Allzu klare Interpretationsanweisungen würden den ironischen Charakter der Äußerung zerstören oder doch deren Wirkung schmälern. ${ }^{3}$ Damit sehen wir uns mit einem eigenartigen Phänomen konfrontiert: in einem derart auf Eindeutigkeit ausgerichteten Interaktionssystem wie dem $\mathrm{Ge}$ richtsverfahren begegnen wir einem kommunikativen Format, das anscheinend erst dann seine soziale Wirkung entfaltet, wenn man es kaum noch wahrnimmt; ein Format, das es den Akteuren verunmöglicht, das, was gemeint ist, ausdrücklich anzusprechen, wollen sie nicht seiner Wirkung verlustig gehen.

Offenbar beißen sich gängige linguistische und literaturwissenschaftliche Forschungsstrategien an der Ironie die Zähne aus. Ein neuerer Übersichtsband (Lapp 1992: 93) kommt jedenfalls zu dem Schluß, daß die linguistische Analyse allein niemals voraussagen könne, was genau eine Äußerung ironisch macht. Dies sei von der jeweiligen Situation abhängig. Es bleibt also nichts anderes übrig, als sich Ironie als praktisches Handeln vor Ort, d. h. für uns, seine spezifische Verwendung im Ablauf von Gerichtsverfahren genauer anzusehen. Dies entspricht natürlich exakt der konversationsanalytischen Forschungstrategie (vgl. Heritage 1984; Psathas 1995). Als Konversationsanalytiker beschäftigen wir uns damit, wie die Beteiligten Ironie im Strafverfahren praktisch realisieren, d.h. welcher interaktiven Methodik sie sich dabei bedienen. Hierzu ist zunächst die für Ironie typische $B e$ teiligtenstruktur sowie die kommunikative Struktur der Ironiesequenz zu klären. Dies sollte uns dann in die Lage versetzen genauer bestimmen zu können, welche besonderen interaktiven Funktionen ironische Passagen im Gerichtsverfahren - insbesondere im Zusammenhang mit der Frage der Glaubwürdigkeit - erfüllen.

\footnotetext{
„Here the duality and allusiveness of the humor would be destroyed by attempts to furnish the kind of clear-cut interpretative instructions that might be suitable in certain serious contexts." (Mulkay 1988: 50). Energischer drückt dies noch Hans Castorp in Thomas Mann's "Zauberberg" aus, wenn er bemerkt: „Aber eine Ironie, die 'keinen Augenblick' mißverständlich ist. - was wäre denn das für eine Ironie, frage ich in Gottes Namen, wenn ich schon mitreden soll? Eine Trockenheit und Schulmeisterei wäre sie!“”
} 


\section{Strukturmerkmale ironischen Sprechens}

\subsection{Die Beteiligtenstruktur}

Schriftliche und mündliche Ironisierungen finden wir in recht unterschiedlichen Formen und Kontexten. So gibt es Beispiele für Ironie in der Literatur, im Cabaret und manchmal auch in wissenschaftlichen Abhandlungen. Die uns interessierende mündliche Ironisierung in Hauptverhandlungen von Strafverfahren weist bestimmte typische Merkmale hinsichtlich der Beteiligtenstruktur sowie der sequentiellen Aspekte ihrer Realisierung auf.

Zunächst fällt auf, daß nur bestimmte Personen Zielscheibe ironischer Bemerkungen werden. Wir haben keine Beispiele dafür gefunden, in denen Sachverständige oder gar Richter ironisiert werden. ${ }^{4}$ Ironische Bemerkungen richten sich vor allem auf Angeklagte und Zeugen; sie sind die primären Objekte der Ironisierung. Dabei fällt auf, daß „neutrale Zeugen“ und von vorneherein geständige Angeklagte so gut wie nie ironisiert werden. Andererseits finden sich keine Beispiele, in denen Zeugen oder Angeklagte andere Verfahrensbeteiligte ironisieren, d.h. als Subjekt der Ironisierung auftreten. Dafür kommen offensichtlich nur die juristischen Hauptakteure, also Richter, Staatsanwälte und Verteidiger in Frage. ${ }^{5}$

Es gibt noch eine dritte in diesem Zusammenhang relevante Teilnehmerkategorie, nämlich die des Ironiepublikums. ${ }^{6}$ Die Zusammensetzung des Ironiepublikums wechselt je nachdem, welcher der ju-

\footnotetext{
${ }^{4}$ Das heißt nicht, daß Fälle einer Ironisierung der Richter durch Angeklagte oder Zeugen nicht vorkommen könnten. Ihre interaktionsstrukturell fundierte Unwahrscheinlichkeit läßt solche Vorkommnisse jedoch zu außerordentlich bemerkenswerten Ereignissen werden. Manche dieser 'Übergriffe' erlangen sogar über den Gerichtsbereich hinaus an Berühmtheit. Man denke an die Szene vor dem Kammergericht in Moabit, in der Fritz Teufel auf die Aufforderung des Richters: „Erheben Sie sich zu Ehren des Gerichtes, Angeklagter!“" mit: „Wenn es der Wahrheitsfindung dient!" reagierte.

"Es gibt sicherlich auch gelegentlich ironische Seitenhiebe in den Plädoyers der Staatsanwälte und Verteidiger auf Art und Stringenz der Ausführungen der jeweils anderen Seite. Dies ist aber ein besonderer Verwendungszusammenhang der Ironie im Verfahren, den wir hier unberücksichtigt lassen.

${ }^{n}$ In der einschlägigen linguistischen Diskussion ist umstritten, ob diese Teilnehmerkategorie für Ironie konstitutiv ist. Der bekannteste Vertreter des ,triadischen Ironiemodells" ist Weinrich (1966). Dezidiert kritisch demgegenüber Lapp (1992).
}

ristischen Akteure die Angeklagten bzw. Zeugen ironisiert (hinzukommen natürlich noch die anderen Anwesenden im Gerichtssaal). Das Besondere der gerichtlichen Kommunikation besteht darin, daß das Ironiepublikum zwar anwesend ist, in aller Regel aber keine offenen Reaktionen (wie Lachen, Kichern, Mißfallenskundgebungen) auf Ironisierungen zeigt. Auch die Ironisierer selbst halten sich diesbezüglich sehr zurück. ${ }^{7}$

Der Hang zu ironischen Bemerkungen hat sicher auch etwas mit dem jeweiligen persönlichen Stil einzelner Ironiesubjekte zu tun. Allerdings kann sich jedweder persönliche Stil nur situationsbezogen, d.h. mit Rücksicht auf den sequentiellen, thematischen, lexikalischen und kategorialen Kontext entfalten. Ironisierungen kommen dementsprechend keineswegs aus heiterem Himmel. Wir finden sie bevorzugt an bestimmten Orten im Verfahrensablauf plaziert. Äußerungen von Zeugen oder Angeklagten werden nur dann ironisiert, wenn bereits vorher Hinweise auf eine mögliche Unglaubwürdigkeit dieser Personen interaktiv herausgearbeitet wurden. Es überrascht von daher nicht, wenn wir Ironisierungen bei den nicht geständigen Angeklagten fast immer antreffen. Demgegenüber gibt es zumindest in unserem Material keine Ironisierung von Zeugen, deren Glaubwürdigkeit als gegeben angesehen oder zumindest nicht schon vorher erkennbar angezweifelt wird.

\subsection{Sequentielle Aspekte der Ironie}

Es reicht nicht aus, wenn die Unglaubwürdigkeit der betreffenden Personen (oder die Unglaubhaf-

\footnotetext{
${ }^{7}$ Welche eminente Darstellungsdisziplin die Beteiligten diesbezüglich an den Tag legen können, zeigt eine Episode, deren Schilderung wir Erving Goffman (1980: 82 f.) verdanken. Es geht in diesem Fall um eine Anklage wegen 'unzüchtiger Aufführung':

„'Diese Darbietung ist Theater hoher Qualität' verkündete Benich (Bruces Anwalt), ehe er auf den Knopf drückte, um die Aufnahme abzuspielen. 'Ich möchte beantragen, daß dem Publikum erlaubt wird, auf die Komik natürlich zu reagieren. Alles andere wäre nicht menschlich.' Richter Horn unterbrach Benich. 'Wir haben hier kein Theater und keine Aufführ ung. Ich gestatte nichts derartiges', entgegnete der Richter. Dann wandte sich Richter Horn an die Zuhörer in dem überfüllten Gerichtssaal und sagte: 'Ich möchte Sie ermahnen, sich bezüglich jeglicher Gefühle zu beherrschen, die in Ihnen aufsteigen mögen'. Diese Ermahnung wurde mit feierlichem Ernst aufgenommen und nicht anders, so zeigte sich, auch die Vorführung. Keiner lachte und nur wenige Anwesende zeigten auch nur die Andeutung eines Lächelns, als die Ausschnitte aus Lenn Bruces humorvollen Darbietungen abliefen."
} 
tigkeit ihrer Aussagen) in der Anklageschrift oder in Unterlagen aus einem vorherigen Verfahren in Frage gestellt worden ist. Die Ironisierbarkeit einer Person muß vor Ort, ,hergestellt" werden. Für ihre Herstellung ist nicht der subjektive Zweifel der Ironiesubjekte, sondern die interaktive Dynamik der tatsächlichen Bezweiflungsaktivitäten vor Gericht entscheidend (,sozialer Zweifel“). Konkret bedeutet dies, daß Ironisierungen erst in späteren Abschnitten der Vernehmung bzw. am Ende der Behandlung eines bestimmten Vernehmungsthemas auftreten. Immer sind ihnen Bezweiflungsaktivitäten in der aktuellen Prozeßsituation vorangegangen. ${ }^{8}$

Auch wenn man auf die Ebene einzelner Redezüge heruntergeht, zeigen Ironisierungsaktivitäten eine bestimmte sequentielle Plazierung. Um interaktiv zu gelingen, muß die Ironisierung im richtigen Moment erfolgen und dabei auf situativ-lokale Phänomene ,schlagfertig" Bezug nehmen. Zunächst fällt auf, daß der Ironisierende immer auf eine andere Aussage, nämlich auf die des späteren Ironisierungsobjektes, reagiert. Typischerweise sind ironisierende Äußerungen somit zweite $\ddot{A} u$ ßerungen, d.h. sie werden als Reaktionen auf einen früheren Redezug einer anderen Person kenntlich gemacht. Dabei handelt es sich praktisch in allen Fällen um den unmittelbar vorhergehenden Redezug der betreffenden Person. Ironische Äußerungen können nur für sich selbst nicht bestehen. ${ }^{9}$ Dies ist ein weiterer Beleg dafür, warum eine rein sprechakttheoretisch argumentierende Konzeption von Ironie problematisch ist. Erst ihre angemessene sequentielle Einbettung läßt eine potentiell ironische Äußerung zu einer wirklichen Ironie werden.

Ironische Attacken brauchen offensichtlich „Aufhänger", wobei der Aufhänger vom Objekt der Ironie geliefert werden muß. Um interaktiv zu gelingen, muß die Ironisierung im richtigen Moment

${ }^{8}$ Die Notwendigkeit, Zweifel in der Gerichtssituation selbst zu plazieren, führen wir also nicht auf den bekannten strafprozessualen Grundsatz der „Mündlichkeit“ zurück (den die juristische Literatur in den Vordergrund stellt), sondern auf die interaktive Struktur und Dynamik von Bezweifungsaktivitäten.

${ }^{4}$ Ähnlich verhält sich dies bei Witzen. Ohne eine entsprechende Einbettung einer Witzerzählung in die laufende Interaktionsepisode kann sich deren potentielle Witzigkeit nicht realisieren. Allerdings wird bei Witzen diese Eingebettetheit in einer vorgeschalteten Eingangssequenz interaktiv etabliert (Sacks 1975; Wolff 1993). Ironisierungen sind von daher interaktiv riskantere Unternehmungen. erfolgen und dabei auf situativ-lokale Phänomene schlagfertig Bezug nehmen. ${ }^{10}$ Die Zielscheibe der Ironie ist die Person, die diese letzte Äußerung getan hat und nicht jemand, der oder die in dieser Äußerung angesprochen wurde. ${ }^{11}$

Diese allgemeinen Beobachtungen möchten wir anhand unseres empirischen Materials noch weiter präzisieren. Unser erstes Beispiel stammt aus einem Verfahren wegen Meineids und versuchter Strafvereitelung.

\section{Ausschnitt 1:}

((Dem Angeklagten Bracke wird vorgeworfen, zugunsten seines Freundes Tupalla in einem anderen Prozeß wegen Trunkenheit im Straßenverkehr eine falsche Aussage unter Eid sowie eine uneidliche Falschaussage gemacht zu haben. In dem jetzigen Verfahren hatte Tupalla bereits als Zeuge ausgesagt, Bracke habe am Steuer gesessen. Er selbst habe auf dem Beifahrersitz mit zurückgedrehter Lehne vor sich hingedöst, während sie von einem Polizeiwagen verfolgt wurden. Im Transkriptionsausschnitt geht es um die Frage, ob die Polizeibeamten zweifelsfrei erkennen konnten, daß nicht Bracke, sondern Tupalla am Steuer $\mathbf{s a B}))^{12}$
$1 \mathrm{ST}$
ja also (0.2) da hab ich an die beidn noch ma eine Frage.
2 RI
3 ST
4
5 ST
6
ja:.
$=$ dieselbe praktisch.
(2.6)
un zwar bezieht sich das nämlich darauf, $(0.8)$ wie die beiden Herrn, (0.4) sich: (1.0) im Pro- fil ähnlich sehn, und: dh (0.4)

${ }^{10} \mathrm{Vgl}$. die analogen Beobachtungen beim „Aufziehen“ (Drew 1987) und „Frotzeln“ (Günther 1994).

11 Letzteres würde im übrigen den Übergang von der Ironie zum Klatsch bedeuten, einer kommunikativen Gattung, die durch das Strafverfahrensrecht in foro implizit zumindest ausgeschlossen wird.

12 Abkürzungen: RI (Richter), ST (Staatsanwalt), VT (Verteidiger), ZE (Zeuge), AN (Angeklagter)

Transkriptionszeichen

hh hörbares Einatmen

?, schwach steigende Intonation

(4.5) Pause von 4,5 Sekunden

(.) kurzes Absetzen

( ) unverständliche Äußerung

(müssen) undeutlich, vermuteter Wortlaut

(( Rascheln)) Kommentierungen des Transkribenten

Fallend-steigende Intonation, die die

Fortsetzung der Äußerung anzeigt.

${ }^{\circ}$ nein ${ }^{\circ} \quad$ leise gesprochen

Emil betont

NEIN größere Lautstärke

$=\quad$ schneller Anschluß

lï: ghe Dehnung des vorhergehenden Lautes

ich nich. stark fallende Intonation 
wie sie von vorne, $(0.4)$ oder auch so wenn se sich bewegn,

(.) welchn Eindruck sie machn da $\sin (d)$ se sich gar nich mehr so ähnlich,

(1.0)

ne:. also jetz wenn ich (se) vor mir sehe, (0.6) aber da sieht ma ma wieder was:: e:: ma muß die Leute sich::

${ }^{\circ} \mathrm{ja},{ }^{\circ}$

(0.4)

bewegn sehn. ma muß sie

(0.2)

(n)ja

ein

(er) im Auto ham wir doch gesessn un ham (e) umgel(a)

grade das wollt ich jetzt die beidn Herrn noch ma fragen das könn wer alles klärn Herr ((langsam, gewichtig)) Tup: alla hh ((abgehackt $))$ so: dann e:

(0.2)

(( schneller, schärfer werdend)) sie kön das ja im übrign gar nich wissen. sie war $n$ ja abgetaucht; sie hattn ja ihrn Rücksitz zurückgestellt un ham die Polizeibeamtn gar nich wahrgenomn; nech, nur noch ma um Ihnn Sie daran zu erinnern, welche Geschichte Sie hier vertretn müssn.

(( Geräusch)) (0.4)

Der Zeuge ( $\mathrm{ZE}$ ) ist in einem vorherigen Verfahren wegen Trunkenheit im Straßenverkehr verurteilt worden; seine Aussagen (und die des jetzigen Angeklagten, der damals als Zeuge auftrat) wurden damals ausdrücklich als unglaubwürdig eingeschätzt. In einem Berufungsverfahren vor dem Landgericht wurde dieses Urteil bestätigt. Dies läßt die Glaubwürdigkeit des Zeugen in diesem Verfahren als problematisch erscheinen. Auch hat der Richter im bisherigen Verlauf der Vernehmung verschiedentlich Zweifel an den Aussagen dieses Zeugen ausgedrückt. ${ }^{13}$ Von daher ist er von

${ }^{13}$ Die interaktive Problematisierung von Aussagen scheint, wie unsere bisherigen Studien ergeben haben, stufenweise zu erfolgen. Am Anfang stehen bloße Nachfragen, dann folgen Fragen. die mit Ausdrücken wie „denn“, "und wieso" und ,ja aber" versehen sind; dann kommen Hinweise auf Widersprüche, Vorhalte und der Rekurs auf vermeintliche Motivlagen. Am Ende dieser Kette stehen dann Konfrontationen mit der Gegenversion und schließlich Ironien und ironische Vorhalte. Obwoh! keinesfalls seiner sozialen Kategorisierbarkeit als „unglaubwürdig" her bereits ein mögliches Bezugsobjekt ironischer Bemerkungen. In dieser Szene nun setzt der Staatsanwalt (ST) - von gelegentlichen $\mathrm{Zu}$ stimmungsäußerungen und Ergänzungen der Richterin (RI) begleitet - an, eine Frage zu formulieren, die er später zwei Polizeizeugen stellen möchte. In dieses Gespräch schaltet sich der Zeuge mit dem Versuch einer Richtigstellung ( ,... im Auto ham wir doch gesessen ...") ein (Zeile 20). Diese Äußerung wird vom Staatsanwalt als Unterbrechung seiner eigenen Äußerung und implizit als eine Verletzung der Zeugenrolle durch $\mathrm{ZE}$ markiert (Zeile 21: ,gerade das wollt ich jetzt die beiden Herren noch mal fragen ..."). Nachdem der Einmischungscharakter und die Voreiligkeit der Äußerung des Zeugen noch ein weiteres Mal betont wurde, folgt ab Zeile 27 die eigentliche ironische Bemerkung. Die ironische Attacke wird so zu einer ,spontanen“ Antwort auf eine „Störung“ durch den Zeugen, die nicht als „gewollt“ oder ,hergeholt" erscheint. Hätte der Staatsanwalt später, d.h. nach einem oder mehreren weiteren $\mathrm{Re}$ dezügen seinen Ironisierungsversuch gestartet, wäre die pragmatische Wirkung vermutlich eine ganz andere gewesen. In den meisten Fällen von Ironisierungen in unserem Material wird dieser unmittelbare Situationsbezug, wie hier, durch Ausdrücke wie ,gerade das", ,genau“ oder ,jetzt" zudem ausdrücklich markiert.

Hinsichtlich der Plazierung der lronie kann man an dem eben präsentierten Beispiel noch eine weitere interessante Beobachtung machen.

\section{Ausschnitt 2:}

$1 \mathrm{ZE}$ - (er) im Auto ham wir doch gesessn un ham (e) umgel(a)

2 ST $->$ grade das wollt ich jetzt die beidn Herrn noch ma fragen das könn wer alles klärn

3 Herr ((langsam, gewichtig)) Tup: alla hh

$4 \mathrm{RI} \rightarrow$ ((abgehackt)) so:. dann e:

$5 \quad(0.2)$

6 ST (( schneller, schärfer werdend))

$7 \rightarrow \quad$ sie kön das ja im übrign gar nich wissen.

8 sie war $\mathrm{n}$ ja abgetaucht; sie hattn ja

alle diese Formate in einem Fall bzw. in einer thematischen Einheit zum Einsatz kommen, beginnen die Bezweiflungen immer mit "schwächeren“ Formaten und steigern sich dann sukzessive. Typisch ist auch, daß solche Steigerungen der Bezweiflungsintensitäten für jeden thematischen Punkt eigens vorgenommen werden. Auf die Unterschiede, die zwischen der Bezweiflung von Äußerungen der Zeugen und jenen der Angeklagten zu beobachten sind, sind wir in Wolff et al. (1996) ausführlich eingegangen. 
9

10

11

12

13

14

15

$16 \mathrm{RI} \rightarrow$ ihrn Rücksitz zurückgestellt un ham die Polizeibeamtn gar nich wahrgenomn; nech, nur noch ma um Ihnn Sie daran zu erinnern, welche Geschichte Sie hier vertretn müssn. (1.0)

(( Geräusch))

(0.4)

Die ironische Äußerung erfolgt, nachdem die Richterin bereits ihrerseits mit ihrem Beitrag begonnen und durch seine Gestaltung einen - möglichen - Abschluß eines Verfahrensabschnittes sowie einen Themenwechsel (hin zur Befragung der Polizeizeugen; Zeile 4: , so::. dann eh“) eingeleitet oder doch zumindest signalisiert hatte. ${ }^{14}$ Die abgehackte Realisierung ihres Redezuges, die Dehnung der einzelnen Redezugkomponenten (so:, dann; e:) und die kurze Pause eröffnen andererseits geradezu die Möglichkeit von „Einschüben“ oder „Ergänzungen“ durch den Staatsanwalt. Auffällig ist auch, daß die Richterin den ironischen Seitenhieb des Staatsanwalts nicht als Unterbrechung ihrer Äußerung behandelt, indem sie nach einer kurzen Pause von einer Sekunde ihre angefangene Äußerung ohne weiteres weiterführt (Zeile 16), d.h. eine ihr durchaus mögliche, ja sogar von dem ,participation framework" des Gerichtsverfahrens her erwartbare Handlungsoption (nämlich: Monieren einer Unterbrechung) nicht wahrnimmt. Die Plazierung der Ironie erfolgt also an einer Strukturstelle, an der der Ablauf des Verfahrens momentan irritiert ist und an der Vorgänge, die eigentlich nicht ins Verfahren gehören, toleriert werden können.

Wir finden somit folgende kommunikative Grundstruktur ironischer Episoden: Grundsätzlich folgt die Ironie auf eine Äußerung des später Ironisierten, die ihrerseits als Einwand, Gegenargument, Behauptung, Klarstellung, Unterbrechung u.ä. formuliert ist und/oder vom Ironisierer retrospektiv als solche markiert wird. Ironische Bemerkungen setzen bevorzugt an vermeintlichen „Vergehen“ gegen das Verfahrenssystem und die darin herrschenden Unterstellungen an. ${ }^{15}$

\footnotetext{
14 Äußerungen wie „so“ und „dann“ dienen in Mehrpersonengesprächen gewöhnlich als Markierer zur Gesprächsstrukturierung. In dem Maße, in dem immer nur Vertreter einer bestimmten Teilnehmerkategorie sich ihrer bedienen, lassen sie sich als Ausdruck situationsinterner Dominanzverhältnisse verwenden (den Hinweis verdanken wir Christoph Meier).

${ }_{15}$ Die ironische Bemerkung bezieht sich meist direkt auf inhaltliche Aspekte der vorherigen Einlassung. Aber auch zurückliegende Äußerungen und Verhaltensweisen des
}

Es fällt auf, daß unmittelbar nach der Äußerung des Ironiesubjektes in den meisten Fällen eine Schweigephase eintritt. In allen Fällen situativ erfolgreicher Ironie erfolgt nach Abschluß der ironischen Äußerung zunächst eine Pause von 1 Sekunde bis zu 20 Sekunden Länge. Wird auf ein Weiterreden trotz ausdrücklicher Anrede zunächst einmal ganz verzichtet, spricht man von einer „Redezugvakanz" (Bergmann 1982). Das heißt, es bestünde für die angesprochenen Zeugen oder Angeklagten an dieser Stelle nicht nur die Möglichlichkeit einer Reaktion, sondern - insbesondere wenn die ironische Attacke in Frageform vorgetragen wird - auch eine entsprechende ,konditionelle Relevanz" dahingehend. Angeklagte wie Zeugen sind gesprächsstrukturell besonders verletzlich für ironische Attacken, weil eine etwaige Gegenironie bzw. eine metasprachliche Beschwerde dagegen nicht zu den kategoriegebundenen Aktivitäten von Zeugen passen. ${ }^{16}$ Typisch für gerichtliche Befragungen ist, daß alle ihre Abschnitte so strukturiert sind, daß sich die Zeugen (noch mehr als die Angeklagten) in der Situation der Reagierenden befinden. Das, was sie zur gerichtlichen Kommunikation beitragen wollen, können sie legitimerweise nur im Rahmen bestimmter Äußerungsformen und -gelegenheiten tun: nämlich nach einer Initiative des Richters und in der Form der gesprächstrukturell passenden Reaktion auf eine derartige Initiative (dies wären vornehmlich Antworten und Sachverhaltsschilderungen). Versuche der Kommentierung bzw. der Zurückweisung von ironischen Attacken auf einer metasprachlichen Ebene sind strukturell dispräferiert und damit interaktiv besonders aufwendig. Da weder das Publikum noch die ironisierte Person auf ironische Bemerkungen als solche reagieren, bekommen ironische Episoden einen eigenartig insulären Charakter im Situationsablauf.

Ironieobjekts können unter bestimmten Umständen ironisch aufgespießt werden. So kann etwa ein Staatsanwalt einen früheren Gesprächsausschnitt (z. B. aus der richterlichen Vernehmung) noch einmal darstellen und daran dann die eigene ironische Bemerkung anschließen. (z. B.: .jetzt hat die Frau Vorsitzende nachgefragt, weil ihr das spanisch vorkam ... und da fiel Ihn: ein, daß Ihre Erinnerung doch da doch etwas nachgelassen hat ...").

16 Vom Zeugen wird erwartet, daß er sich „ernsthaft bemüht", eine vollständige und richtige Aussage zu machen. Dies ergibt sich u.a. aus der Analyse von Darstellungen glaubhafter Zeugenaussagen in schriftlichen Urteilsgründen. Zum konversationsanalytischen Konzept der ,category-bound-activities"vgl. Atkinson/Drew (1979: 122f.). 


\subsection{Merkmale ironischer Äußerungen}

Schauen wir uns nun die ironische Äußerung im engeren Sinne an. Hier stellt sich die Frage: Was macht sie als ,,ironisch gemeint" oder zumindest als ,ironiefähig“" erkennbar? Einige Beobachtungen lassen sich am folgenden Ausschnitt unseres Beispiels machen.
Ausschnitt 3:
$1 \mathrm{ST} \rightarrow>$ grade das wollt ich jetzt die beidn Herrn noch 2 Herr ((langsam, gewichtig)) Tup: alla hh
3 RI -> ((abgehackt))
4 so:. dann e:
$5 \quad(0.2)$
6 ST (( schneller, schärfer werdend $))$
$7->\quad$ sie kön das ja im übrign gar nich wissen.
8 sie war $n$ ja abgetaucht: sie hattn ja
9 ihrn Rücksitz zurückgestellt un ham die
10 Polizeibeamtn gar nich wahrgenomn; nech,
nur noch ma um Ihnn Sie daran zu erinnern, welche Geschichte Sie hier vertretn müssn.

Der Staatsanwalt geht offenbar davon aus, daß der Zeuge etwas behauptet hat, was er nach seiner vorherigen Darstellung, wonach er vor sich hingedöst und die Beamten nicht wahrgenommen habe, gar nicht wissen kann. Wie hier beziehen sich viele ironische Bemerkungen auf Widersprüche. Bei dieser Bezugnahme fällt die übertriebene „Fürsorglichkeit" des Staatsanwalts auf. Nimmt man seine ÄuBerung wörtlich, dann "hilft" er dem Zeugen dabei, eine bestimmte Geschichte vertreten zu können. Eine solche Interpretation des Sinns der ÄuBerung des Staatsanwalts kann aber nicht richtig sein. Wie jeder weiß, besteht die Pflicht des Zeugen im Gegenteil darin, die Wahrheit mitzuteilen, d.h. eben keine Geschichte zu erzählen. Hinzukommt, daß der Staatsanwalt gleichsam zum ,gegnerischen Team" gehört, aber an dieser Stelle so tut, als ob dies gar nicht der Fall sei und er sich unvoreingenommen, ja sogar helfend an den Zeugen wenden würde. Die ironische Pointe, also die Auflösung dieses Widerspruchs, läuft darauf hinaus, daß der Zeuge eben nicht die Wahrheit sagt, sondern versucht eine (erfundene) Geschichte zu vertreten.

Neben Aussagewidersprïchen wird in ironischen Bemerkungen vor allem auch die Unwahrscheinlichkeit einer Darstellung aufs Korn genommen. Die Konstatierung der statistischen Unwahrscheinlichkeit einer Darstellung oder Behauptung reicht freilich für eine grundlegende Bezweiflung der Glaubhaftigkeit der Aussage bzw. der Glaubwürdigkeit der jeweiligen Person ebensowenig aus wie die Kontrastierung der Aussage mit bestimmten Normalformerwartungen. Eine sozial relevante Unwahrscheinlichkeit muß zunächst in der Situation etabliert werden. Die Ironisierung scheint ein kommunikatives Instrument zu sein, um aus einer bloß unwahrscheinlichen eine zweifelhafte Aussage zu machen, die auf Unglaubwürdigkeit schließen läßt. ${ }^{17}$ Ein Aspekt ironischer Äußerungen besteht entsprechend darin, vorherige Darstellungen der Ironieobjekte noch einmal aufzugreifen und sie dabei erkennbar zu überziehen. ${ }^{18}$

\section{Ausschnitt 4:}

((Der Zeuge, ein Handelsvertreter, hatte unmittelbar vorher behauptet, dem Angeklagten, einem Drogenabhängigen, 5000 Mark für ein geschäftliches Unternehmen geliehen zu haben, und zwar ohne genau zu wissen, wofür das Geld bestimmt war. Noch dazu hatte er selbst einen Teil dieses Geldes von anderen Personen leihen müssen))

\begin{tabular}{|c|c|}
\hline $1 \mathrm{RI}$ & $\begin{array}{l}\text { und sie haben sich quasi anderen gegenïber } \\
\text { auch }\end{array}$ \\
\hline 2 & $\begin{array}{l}\text { noch mal verpflichtet um ihm das Geld zu } \\
\text { ihm das Geld zu }\end{array}$ \\
\hline 3 & besorgen. \\
\hline $4 \mathrm{ZE}$ & richtig. \\
\hline $5 \mathrm{RI}->$ & $\begin{array}{l}\text { dafür gibt } s \text { keine andere Erklärung als daß } \\
\text { sie ihm das: }\end{array}$ \\
\hline 6 & (1.6) \\
\hline 7 & auf $\operatorname{sein}(e)$ \\
\hline 8 & $(1.0)$ \\
\hline 9 & seine nette Art hin \\
\hline 0 & $(0.4)$ \\
\hline 11 & zur Verfügung stellen. \\
\hline 12 & (3.0) \\
\hline $13 \mathrm{ZE}$ & $\begin{array}{l}{ }^{\circ}(\text { hh })^{\circ} \text { e: hff: : ja. w wo hätt er s Geld sonst } \\
\text { herkriegen; }\end{array}$ \\
\hline
\end{tabular}

Wenn jemand, noch dazu, wenn er kaufmännisch tätig ist, einer anderen Person lediglich ,auf seine nette Art hin" 5000 Mark leiht, wäre dies außergewöhnlich naiv. Die Bemerkung des Richters läuft darauf hinaus, daß der Zeuge dazu eingeladen wird, sich selbst als naiv zu bezeichnen, ${ }^{19}$ um der anderen Interpretationsmöglichkeit (,unglaubwürdig") zu entgehen. Ein wesentliches Merkmal der ironisierenden Äußerungen besteht also darin,

17 Wir sprechen hier nur mehr von Glaubwürdigkeit, weil die Ironisierung sich nie nur auf die Aussage, sondern immer auch auf deren Urheber bezieht.

${ }_{18}$ Analog der Überzogenheit der angetragenen „Fürsorglichkeit" im Ausschnitt 3.

${ }^{14}$ Denkbar wären grundsätzlich natürlich auch andere Selbstkategorisierungen: der Zeuge könnte sich beispielsweise als "Bisexueller“ outen, der auf den Angeklagten „abgefahren“ sei. Der zusätzliche Erklärungsaufwand wäre allerdings erheblich. 
daß in ihnen Kontraste enthalten sind, die sich nicht auflösen lassen ohne bestimmte Normalitätsannahmen zu tangieren. Dies erschwert es außerordentlich, auf ironische Fragen oder Bemerkungen direkt zu reagieren. Geschickt gewählte Kontraste bringen das Ironieobjekt in eine Art von Doppelbindung: egal, wie es reagiert - seine Reaktion ist problematisch. Reagiert es so, als ob nichts gewesen wäre, demonstriert es seine Naivität oder bekennt gar seine soziale Inkompetenz. Reagiert es nicht, so ist ihm diese Nicht-Reaktion als ,offizielles Fehlen" zurechenbar (dies ist der häufigere Fall!). Wenn es andererseits die Zumutung anspricht, die in der ironischen Äußerung impliziert ist, überschreitet es das für sie im Aktivitätstyp Gerichtsverfahren strukturell vorgesehene Handlungsrepertoire.

Kontraste und Übertreibungen kommen zwar in ironischen Äußerungen häufig vor; sie sind aber für sich genommen keine eindeutigen Indikatoren für Ironie; vor allem keine Indikatoren, die allein für Ironie spezifisch wären. Es hat den Anschein, als wenn es unmöglich wäre, nur anhand von inhaltlichsemantischen Merkmalen bestimmen zu wollen, ob eine Äußerung ironisch oder ernsthaft gemeint war. Die Rede von „,ironischen Sprechakten“ ist demnach irreführend. Angesichts dessen hat man sich ja auch in der linguistischen und literaturwissenschaftlichen Diskussion bemüht herauszufinden, ob die Mitglieder wenigstens auf gewisse formale Merkmale von Aussagen zurückgreifen können, um ironische Bemerkungen zu identifizieren. Es gibt in der Tat eine ganze Reihe von Kandidaten für derartige Ironiesignale: u.a. werden mimische, gestische und intonatorische Modulationen wie Augenzwinkern, Räuspern, eine emphatische Stimme oder die Häufung bombastischer Ausdrücke, gewagte Metaphern, überlange Sätze, Wortwiederholungen, "hörbare" Anführungszeichen als Kandidaten genannt. Alles dies kommt auch in unserem Material vor, ohne daß dabei freilich eine durchgängige Systematik erkennbar wäre.

Der obige Ausschnitt 3 zeigt z. B. zwei, auf der averbalen Ebene relativ häufig zu beobachtende Merkmale ironischer Bemerkungen. An diesen Äußerungen fällt oft eine gegenüber vorhergehenden Abschnitten veränderte Tonlage (in unseren Beispielen meist lauter) und Sprechgeschwindigkeit auf. Hinsichtlich der Sprechgeschwindigkeit konnten wir keine eindeutige Richtung beobachten: zwar steigert diese sich in der Mehrzahl der Fälle, es kommen aber auch auffällige Verzögerungen und Rhythmisierungen (Wort-für-Wort-Sprechen) vor. Ähnlich bei der Tonlage: in unserem Material steigert sie sich meist; gelegentlich aber finden wir aber auch im Vergleich zum Vorherigen leisere Ironisierungen.
Selbst wenn wir demnach keine eindeutigen Markierer für Ironie identifizieren können, so ergibt sich doch eine weiterführende Beobachtung: Alle erwähnten Ironiesignale haben nämlich gemeinsam, daß sie dazu beitragen, die betreffende Äußerung aus dem Verlauf der übrigen Interaktion wahrnehmbar herauszuheben. In dieselbe Richtung weist der phasenweise Gebrauch von Dialekt- und Slang-Varianten (,könn' wer"). Die einzige Regel, die sich demnach hinsichtlich der Markierung ironischer Äußerungen angeben läßt ist, $d a \beta$ sie markiert sind, und daß den potentiellen Ironikern um dies zu erreichen ein breites Repertoire von Möglichkeiten zur Verfügung steht. Markiert wird nicht der ironische Charakter der Äußerung (dies würde ein eindeutiges Entsprechungsverhältnis von Bedeutung und Äußerung voraussetzen), sondern vielmehr der Umstand ihrer Besonderheit.

Dafür spricht noch eine weitere Beobachtung: Es gibt eine Klasse von konversationellen Phänomen, die zwar in den üblichen Listen von Ironiesignalen fehlt, die sich aber zumindest in unserem Material fast durchgängig findet: Gemeint sind Ausdrücke wie ,nur noch mal", ,im übrigen“, „nur um", „möglicherweise“, „eigentlich“, ,ich muß leider", „es gibt keine andere Erklärung" u.ä.. Derartige sprachliche Phänomene werden in der linguistischen Pragmatik als "hedges" gezeichnet. Unter ,hedges" versteht man ein Partikel, ein Wort oder eine Floskel,

"that modifies the degree of membership of a predicate or noun phrase in a set; it says of that membership that it is partial, or true only in certain respects, or that it is more true and complete than perhaps might be expected." (Brown/Levinson 1987: 145)

„Hedges" haben insbesondere die Funktion, potentiell unhöfliche und unkooperative Implikationen von Äußerungen abzumildern. Durch ihre Verwendung wird einerseits die Außergewöhnlichkeit (und das Bedrohungspotential!) der Ironie markiert, andererseits aber einer entsprechenden feindseligen oder entrüsteten Reaktion darauf vorgebaut.

Somit können wir als Zwischenresümee festhalten, daß zwar sprachliche und außersprachliche Signale dem Hörer ein ironisches Verständnis erleichtern; die Verwendung dieser Signale sich aber als sehr uneinheitlich erweist. Die linguistische Analyse allein kann deshalb niemals voraussagen, ob eine Äußerung ironisch ,ist“. Letztlich wird erst durch die Reaktion der Rezipienten darüber entschieden, ob ein Ironisierungsversuch ,glückt" oder „daneben geht“. Eine Konversationsanalyse der 
Ironie wird sich (im Gegensatz zur sprechakttheoretischen Zugangsweise) deshalb nicht auf die Analyse der Äußerungen des Ironiesubjekts beschränken. Sie muß zu rekonstruieren versuchen, wie die Gesprächsteilnehmer selbst diese Äußerung analysieren und ihrem Sinn damit die Eigenschaft einer ,sozialen Tatsache“ verleihen.

\subsection{Reaktionen der Ironisierten}

Sequenzanalytisch gesehen würde der Versuch einer Ironisierung immer dann mißlingen, wenn alle Rezipienten durch die Art und Weise ihrer Reaktion zu erkennen geben, da $\beta$ sie die betreffende Äußerung ernst nehmen - ohne die Verspottung zu registrieren. Hier entsteht allerdings in Bezug auf unseren Gegenstand Gerichtsverfahren eine paradoxe Konstellation: Ganz offensichtlich legen sich die verschiedenen Rezipienten besondere $B e$ schränkungen hinsichtlich ihrer Reaktionen auf. In keinem Fall aus unserem Material kommt es zu lautem Lachen oder ähnlichen Kundgaben der Belustigung.

Dies ist insoweit nicht überraschend, als anerkanntermaBen die besondere Eleganz ironischer Kommunikation nicht nur darin besteht, daß sie gleichsam beiläufig daherkommt, sondern auch, daß sie in ähnlich beiläufiger Weise von den Beteiligten goutiert wird. Gerade die "feine" und treffsichere Ironie scheint keine allzu deutlichen Reaktionen der anderen Beteiligten zu vertragen. Beide Seiten müssen sich also in ihrer Zurückhaltung ergänzen. Die Gefahr gerade für die ,feine“ Ironie liegt allerdings darin, da B sowohl die ironisierende Äußerung wie die Reaktionen der Rezipienten aufgrund ihres geringen Explizitheitsgrades leicht übersehen oder mißverstanden werden können. ${ }^{20}$

Selbst wenn die Ironie und damit einhergehende massive Infragestellung eines Zeugen bzw. Angeklagten für alle Beteiligte subjektiv völlig eindeutig sein mag, so sind doch ihre Reaktionsmöglichkeiten in der gerichtlichen Interaktionssituation grundsätzlich, wenn auch in unterschiedlicher Weise eingeschränkt. Wir haben, wie schon erwähnt, keinen Fall beobachtet, in dem sich einer der Beteiligten, vor allem nicht die ironisierte Person selbst, ausdrücklich auf den Sachverhalt der Ironisierung bezieht (etwa in Gestalt einer Beschwerde über die situative Unangemessenheit humoriger Aussagen). ${ }^{21}$ Offiziell bleibt die ironische Komponente

\footnotetext{
20 Nach Umberto Eco (1984) besteht das Schöne (und die Gefahr) an der Ironie darin, daß es immer jemanden gibt. der das ironisch Gesagte ernst nimmt.

21 Diese Zurückhaltung gilt nur für das gerichtliche Interaktionssystem. In Interviews über die entsprechenden Verhandlungen erzählten die Beteiligten sehr wohl
}

der Kommunikation "ex-kommuniziert". Von allen Beteiligten wird so getan, als ob die Situation ihren ernsthaften Charakter zu keiner Zeit verloren hätte. Man könnte diesbezüglich die Interaktionsordnung des Gerichts als einen partiell geschlossenen „Bewußtheitskontext" bezeichnen. Die Betroffenen wissen in den meisten Fällen sehr wohl, welches Spiel (mit ihnen) hier getrieben wird, können dies aber aufgrund der ,interaction order" der gerichtlichen Vernehmung von sich aus nicht zum Ausdruck zu bringen. ${ }^{22}$

Die besondere „Kooperativität“ der Ironieobjekte, also der eigentlich Leidtragenden ironischer Attacken, drückt sich darin aus, daß sie mit Redezugvakanzen oder ,ernstgemeinten“ Rechtfertigungen reagieren. Der folgende Ausschnitt zeigt ein Beispiel für diese eigenartige „Kooperation“ durch Schweigen. Zu seinem Verständnis ist daran zu erinnern, daß die Äußerungen der Ironiesubjekte in vielfältiger Weise als besondere und damit als potentiell ironische markiert sind. Diese Herausgehobenheit wird dadurch noch verstärkt, daß nach der eigentlichen ironischen Bemerkung von der ironisierenden Person keine weiteren Aussagen zu diesem Thema folgen, statt dessen eine Pause eintritt und von einem anderen Beteiligten aus dem Ironiepublikum die Interaktion ohne weiteren Kommentar auf ein neues Thema ausgerichtet wird. ${ }^{23}$

\section{Ausschnitt 5:}

$\begin{array}{lll}1 & \text { VT } & \text { heut sagen sie klipp und klar } \\ 2 & & \text { keinesfalls der Türke. } \\ 3 & (0.2) \\ 4 & \text { jetzt könn mer uns was aussuchen. } \\ 5-> & (13.6) \\ 6 ? ? & ((\text { Atmen, Geräusche })) \\ 7-> & (7) \\ 8 \text { RI } & \text { ham Sie bei der Polizei gesagt es sei dieselbe } \\ 9 & \text { Person } \\ & \text { gewesen? }\end{array}$

über das Vorkommen wie über die Treffsicherheit ironischer Bemerkungen. Auch wurde hier die motionale Beteiligung (Wut, Entrüstung oder Belustigung), sowie etwaige dadurch motivierte prozeßstrategische Konsequenzen (Stellen von Befangenheitsanträgen, Verzicht auf die eigene Befragung des Zeugen oder Angeklagten, 'Fallenlassen' des ironisierten Zeugen) deutlich zum Ausdruck gebracht.

22 In der Terminologie von Glaser/Strauss entspräche dem in etwa der "pretense awareness context". Dieser ist ,a modification of the open one: both interactants are fully aware but pretent not to be." (Glaser/Strauss 1964: 670) 23 In Fällen, in denen der Richter eine ironische Attacke startet, ist in der Regel er es, der den Themenwechsel vornimmt. 
Zur interaktiven Wirkung der Äußerung des Verteidigers trägt vor allem auch der Umstand bei, daß dieser darauf verzichtet, die Reaktion des Zeugen einzufordern. ${ }^{24}$ Man kann Pausen in derartigen Konstellationen als interaktive Strategien ansehen, die eingesetzt werden, um für alle Beteiligten erkennbar auf das Fehlen einer angemessenen Reaktion und damit indirekt auf den problematischen Status des Befragten hinzuweisen.

Eine dritte Variante der kooperativen Herstellung von Ironie zeigt das nächste Beispiel. Hier wird der ironische Charakter der Episode ebenso wie die für die ironisierte Person problematischen Implikationen der ironischen Äußerung durch eine längere Schweigephase sowie durch die daran anschließende Reaktion des Zeugen deutlich gemacht. Indem dieser an die Pause eine Rechtfertigung anschließt, akzeptiert und relativiert er (durch die Gegenfrage) den in der ironisierenden Äußerung verkörperten $Z$ weifel.

\section{Ausschnitt 6:}

\begin{tabular}{|c|c|}
\hline $1 \mathrm{RI}$ & $\begin{array}{l}\text { dafür gibt s keine andere Erklärung als daß } \\
\text { sie ihm das: }\end{array}$ \\
\hline 2 & (1.6) \\
\hline 3 & auf $\operatorname{sein}(e)$ \\
\hline 4 & $(1.0)$ \\
\hline 5 & seine nette Art hin \\
\hline 6 & $(0.4)$ \\
\hline 7 & zur Verfügung stellen. \\
\hline 8 & $(3.0)$ \\
\hline $9 \mathrm{ZE}->$ & $\begin{array}{l}{ }^{\circ}(\text { hh })^{\circ} \text { e: hff: : ja. w wo hätt er s Geld sonst } \\
\text { herkriegen: }\end{array}$ \\
\hline
\end{tabular}

Ironische Attacken gelingen keineswegs in jedem Fall. Sie können insbesondere dann ins Leere laufen, wenn das Ironiesubjekt in seinem Redezug mehrere Optionen der Reaktion offenläßt und der Angesprochene von daher seine Mitarbeit an der eigenen Ironisierung ohne unkooperativ zu erscheinen vermeiden kann. Im nachfolgenden Abschnitt bezieht sich der Zeuge in seiner Reaktion nicht auf die unmittelbar vorhergehende ironisierende $\ddot{A} u ß e r u n g$, sondern auf eine Frage, die der Staatsanwalt in seinem Redezug an früherer Stelle gestellt hatte. Dadurch nimmt er dem Ironisie-

\footnotetext{
${ }^{24}$ Nach längeren Redezugvakanzen ist erwartbar, daß der vorherige Sprecher auf die Redezugvakanz verbal reagiert, etwa indem er fragt, ob er verstanden wurde, indem er seinen Beitrag wiederholt oder reformuliert oder indem er das Schweigen kommentiert (vgl. Bergmann 1982). Insbesondere gilt diese Erwartung für ironisierende Richter, da sie im Gerichtsverfahren als primäre Sprecher etabliert sind.
}

rungsversuch viel von seiner Wirkung. ${ }^{25}$ Bei genauerem Hinsehen zeigt sich freilich, daß dieses „Übergehen“ des Ironisierungsversuchs nicht voll ständig gelingt. Die immerhin 1.4 Sekunden währende Pause und die deutlich zögernde Einleitung der Replik (,(nj)a s wird wohl schon so gewesn sein") machen diese als Reaktion auf einen ironischen „Treffer“ hörbar. ${ }^{26}$

\section{Ausschnitt 7:}

$\begin{array}{lll}1 \mathrm{ST} \rightarrow & \text { ( ) (z) muß ich doch noch ma genau fragn. } \\ 2 & \text { wie war s denn nun wirklich. } \\ 3 \mathrm{ZE} & \text { ( ) ja (s) ( } \\ 4 \mathrm{ST} & \text { oder; } \\ 5 & \rightarrow> & \text { kann s gibt s vielleicht noch ne dritte } \\ 6 & & \text { Möglichkeit die in Betracht kommt; die } \\ 7 & & \text { Ihnn möglicherweise noch gar nicht einge }= \\ 8 & \text { falln is.(. } \\ 9 & (0.6) \\ 10 ? ? & \text { ((Räuspern)) } \\ 11 & \text { (0.8) } \\ 12 & \mathrm{ZE}-> & \text { (nj)a s wird wohl schon so gewesn sein (daß) } \\ 13 & \text { ( ) (s) Auto aus der Einfahrt rausfahrn wollte } \\ 14 & \text { damith halt (ds) }\end{array}$

Der „Bewußtheitskontext der Verstellung“ bezieht, wie schon angedeutet, das Ironie-Publikum mit ein. Es entsteht eine Art stillschweigender

${ }^{25}$ In diesem Fall wird die Bezweiflungsphase an dieser Stelle nicht abgeschlossen; statt dessen erfolgt eine weitere Bezweiflungssequenz. Nur geglückte Ironierungsversuche eignen sich offenbar als Abschluß einer themenbezogenen Bezweiflungsphase.

${ }_{26}$ An diesem Beispiel ließen sich umfangreiche Überlegungen hinsichtlich der Reichweite einer konversationsanalytischen Forschungsstrategie anschließen, die hier nicht entfaltet werden können. Nur so viel: Die Schwierigkeit bei der Identifizierung von Ironie in Fällen, bei denen kaum erkennbare Reaktionen auf „Ironiekandidaten“ auftreten, scheint die Konversationsanalytiker als verkappte Positivisten zu entlarven, die nicht in der Lage sind, die alltagssprachliche Kompetenz des Ironie-Überhörens und seine Feststellung zu rekonstruieren. Insoweit sich diese vermeintliche Kompetenz nicht in der Systematik kommunikativer Handlungen manifestiert, ist sie in der Tat einer konversationsanalytischen Zugangsweise nicht zugänglich. Dies erscheint hier insoweit unproblematisch zu sein, als unser Ehrgeiz ja dahingeht, Ironie als soziale Tatsache im Rahmen eines bestimmten Interaktionssystems in ihrer interaktiven Methodizität zu analysieren. Das rigorose Beharren auf dieser ,positivistischen" Position hat den nicht zu unterschätzenden Vorteil, die Grenze zwischen Interaktions- und personalen Systemen bzw. jene zwischen einer soziologischen und ejner psychologischen bzw. phänomenologischen Behandlung von Ironie aufrecht zu erhalten. 
Komplizenschaf ${ }^{27}$ zwischen dem Ironisierer und den Zuhörern. Typisch für diese Komplizenschaft ist, daß sie sich nicht oder nur ansatzweise offen äußert. ${ }^{28}$ Diese Komplizenschaft ist weder ganz verläßlich (aus der Sicht des Ironisierenden, der sich nie ganz der Wirkung seiner Ironie sicher sein kann), noch wirklich einklagbar (aus der Sicht aller Beteiligten) - etwa im Hinblick auf die Wertung der betreffenden Aussage in der Urteilsbegründung. Gleichwohl fällt auf, daß ironische ÄuBerungen durch ihre vielfältigen Markierungen und interaktiven Bestätigungen eine gewisse Prägnanz gewinnen. Zumindest scheinen sich ironisch markierte Äußerungen den Beteiligten in besonderem Maße einzuprägen. Dies zeigt sich etwa darin, daß manche der Äußerungen, die Anlaß zu ironischen Kommentaren gaben, in Plädoyers und in Urteilsbegründungen wieder auftauchen - auch wenn ihre implizite Botschaft in der Situation selbst nicht formuliert worden war. In dem Prozeß, aus dem der gerade zitierte Abschnitt stammt, verweist sowohl der Staatsanwalt in seinem Plädoyer, als auch anknüpfend daran die Richterin in der mündlichen Urteilsbegründung auf die ironisch markierte Stelle und begründet damit ihre Feststellungen über die zweifelhafte Glaubwürdigkeit dieses Zeugen. ${ }^{29}$

\section{Ausschnitt 8:}

ST so wie der Herr Tupalla uns auch an einer anderen Stelle die Unwahrheit erzählt hat. wie ich feststellen muß. das is nämlich als er erzählt hat er hätte geschlafen auf $\mathrm{m}$ Beifahrersitz vor sich hingedöst.

\footnotetext{
27 Auch jene Beteiligten aus dem Publikum, die die Intentionen der ironisierenden Person nicht teilen, tragen, indem sie stillhalten, wenn auch unfreiwillig, zum 'Erfolg' der Ironisierung bei.

${ }^{28}$ Einschränkend ist zu bedenken, daß wir bei unseren Analysen auf Tonbandaufnahmen angewiesen sind. In Beobachtungen haben wir gewisse Reaktionen wie 'vielsagende' Blicke, Lächeln u.ä. bei Ironiesubjekten wie beim Ironiepublikum feststellen können. Allerdings sind diese Reaktionen nicht einheitlich und auch nicht immer gegeben. Für eine strukturelle Analyse der Ironie im Verfahren würden derartige Hinweise auf Ironiesignale deshalb vermutlich auch dann nicht ausreichen, wenn wir auf Videomaterial zurückgreifen könnten.

${ }^{29}$ Ein solches Eingehen auf ironische Passagen während des Prozeßverlaufs findet sich in keinem Fall in den schriftlichen Urteilsgründen (von denen uns etwa 200 vorliegen). Dies verweist wiederum auf die Situationsabhängigkeit der Ironie, auf ihre primär interaktive Funktionalität, sowie darauf, daß mündliche und schriftliche Urteilsbegründungen an unterschiedliche Adressaten gerichtet sind.
}

von den Beamten garnicht viel mitgekriegt dann hat er sich dummerweise nachher verplappert und hat uns erzählt. wie die Beamten reagiert haben als sie ins Fahrzeug rüber gekuckt haben. nun das kann er schwerlich festgestellt haben wenn er betrunken dösend. eh aufm runtergekurbelten. Sitz. der Beifahrerseite gesessen hat. ((betont)) auch das war die Unwahrheit.

\section{Ausschnitt 9:}

RI und der Herr Staatsanwalt hat die Kernpunkte. eh insbesondere in der Aussage des Zeugen Tupalla heute doch ganz klar aufgedeckt. er hat sich dann auch verplappert. und

Die Ironie hat ihren festen interaktions-logischen Ort in der gesamten Dramaturgie der Befragung von Zeugen und Angeklagten. Phasen der $\mathrm{Be}$ zweiflung bzw. von Phasen der Konstruktion von Unglaubwürdigkeit werden häufig nach ironischen Äußerungen abgeschlossen. Praktisch alle Ironisierungen in unserem Material finden sich dermaßen plaziert. Sie fungieren gleichsam als $S c h l u \beta-b z w$. Höhepunkte von Bezweiflungsprozessen. Nach geglückten Ironien erfolgt typischerweise ein mehr oder weniger ausgeprägter Wechsel des thematischen Angriffspunktes und/oder ein Wechsel der befragenden Person. Waren es Staatsanwälte und Verteidiger, welche die Ironie plazierten, so nimmt meist der Richter von sich aus wieder die Befragung in die Hand (etwa durch einen neuen Vorhalt), wie im folgenden Ausschnitt:

\section{Ausschnitt 10:}

((Der Zeuge hatte nach dem polizeilichen Vernehmungsprotokoll ausgeführt, er habe sich mit einem bestimmten Türken in einer Gaststätte getroffen. In dieser Zeugenvernehmung dagegen spricht er davon, er habe sich nicht mit „dem Türken“, sondern mit einer anderen Person getroffen. Darauf spielt der Verteidiger mit seiner Äußerung ,jetzt könn mer uns was aussuchen" an.))

$\begin{array}{lll}1 & \text { ZE } & \text { hab s verstanden } \\ 2 \text { VT } & \text { =in der Aussage steht klipp und klar } \\ 3 \text { ZE } & \text { ( ) (ich sp- ich) } \\ 4 \text { VT } & \text { drin es war der - } \\ 5 \text { ZE } & \text { spreche doch von zwei verschiedenen Perso- } \\ & \text { nen. }\end{array}$

7

8

$9->$

10

$11 ? ?$

12

13 RI -> ham Sie bei der Polizei gesagt es sei dieselbe
Person gewesen? klar keinesfalls der Türke. $(0.2)$

jetzt könn mer uns was aussuchen. (13.6) ((Atmen, Geräusche $))$

$(7)$ 
Wir haben hier den Fall vor uns, daß der Zeuge schweigt und der Verteidiger seine Zeugenbefragung selbst nach mehr als 20 Sekunden Pause nicht wieder aufnimmt. Die Ironie bleibt damit quasi im Raume stehen. Den dadurch sich aufbauenden „Kommunikationsstau“ löst der Richter (als primärer Sprecher des Verfahrens) durch eine versachlichende Frage auf (seine Frage impliziert, daß der Widerspruch auch durch einen Protokollierungsfehler entstanden sein könnte). Indem der Richter nicht einfach das Thema wechselt, sondern es bloß versachlichend verschiebt, nimmt er dem ironischen Effekt des Verteidigerbeitrags ein wenig die Spitze. Das Beispiel zeigt, daß die Prozeßbeteiligten ein unterschiedlich großes Interesse an einer Degradation von Zeugen haben. In diesem Fall geht es um die Glaubwürdigkeit eines Belastungszeugen, von dessen Aussage es abhängen kann, ob der Angeklagte zu einer hohen Freiheits strafe verurteilt wird.

\section{Ironie und Unglaubwürdigkeit}

In der Literatur wird gelegentlich vermutet, die interaktive Funktion ironischer Bemerkungen bestünde primär in der Ausübung sozialer Kontrolle. Insofern mit sozialer Kontrolle jene Prozesse und Mechanismen gemeint sind, die eingesetzt werden, um Gesellschaftsmitglieder zu bestimmten, als positiv oder erwünscht angesehenen Verhaltensweisen zu bewegen, bieten unsere Daten hierfür keine Belege. ${ }^{30}$ Auf ironische Bemerkungen geschieht

\footnotetext{
30 Als Konversationsanalytiker konzentrieren wir uns auf die besondere 'Interaktionsordnung' der Zeugenvernehmung. Dadurch mag der Eindruck entstehen, wir würden Phänomene der Macht ausblenden und damit ein unkritisch-naives Bild der Verfahrensabläufe zeichnen. In der Tat interessieren wir uns nicht für Machtunterschiede und Dominanzphänomene, soweit sie konzeptuell auf einzelne soziale Akteure oder Klassen von Akteuren bezogen werden (im Sinne einer 'Dominanz der Experten'). Unsere analytischen Anstrengungen sind auf strukturelle Arrangements, d.h. auf bestimmte Handlungsformen gerichtet, innerhalb derer sich die Beteiligten zur Verfolgung ihrer strategischen Absichten bewegen müssen. In der jeweiligen Interaktionsordnung lassen sich sicherlich Asymmetrien im Hinblick auf bestimmte Beteiligtenkategorien und die ihren konventionellerweise eingeräumten Handlungsmöglichkeiten ausmachen. $\mathrm{Ob}$ und wie diese strukturellen Asymmetrien tatsächlich umgesetzt und in welcher Weise sie zur Verfolgung strategischer Interessen genutzt werden können, ist Ergebnis praktischen Handelns aller Beteiligten vor Ort. Weil soziale Wirkungen nur innerhalb der betreffenden Handlungsform erreicht werden kön-
}

typischerweise „nichts“. Meist folgt ein Schweigen, und/oder das Ironieobjekt versucht (dann) eine möglichst ernsthafte Bestätigung seiner bisherigen Aussage. In keinem Fall haben wir eine Selbstkorrektur der Ironisierten beobachtet, was bei einem funktionierenden sozialen Kontrollmechnismus zu erwarten gewesen wäre. Angesichts dessen erschiene es uns irreführend, die Funktion von Ironie darin zu sehen, daß durch sie Zeugen oder Angeklagte dazu gebracht werden sollten, die Wahrheit zu sagen oder zumindest doch plausiblere Versionen zu produzieren.

Zutreffender wäre es davon zu sprechen, daß durch Ironie im Strafverfahren Differenz etabliert wird. Differenz zwischen denen, die über die Ironie offen, häufiger aber insgeheim schmunzeln (also die bzw. ein Teil der Zuhörer) und jener Person, die gerade ironisiert wird. Die Ironie übt ihre Wirkung in doppelter Weise aus: sie schafft eine heimliche Gemeinschaft derjenigen, welche die implizite Botschaft auf dem Hintergrund der Verfahrensgeschichte verstehen (dazu gehört oft auch das Ironieobjekt), und konstituiert zugleich eine Differenz zwischen der ironisierten Person und den übrigen „Wissenden“. Die Ironie spielt immer auf ein gemeinsames Hintergrundwissen an (dazu gehört insbesondere die vorherige Befragungsbzw. Bezweiflungssequenz, aber auch der bisherige Prozeßverlauf), dessen Inrechnungstellung für eine adäquate Interpretation der Intention der Ironie notwendig ist. Auf diese gemeinsame Interaktionsgeschichte spielt die Ironie nicht nur an. sie liefert auch deren dramatischen Höhepunkt. Sie tut dies in einer besonders markanten Weise, was dazu beiträgt, daß nicht nur die betreffende Episode im Gruppengedächtnis konserviert wird; auch der gemeinsame Erfahrungsbestand wird bestätigt und bekräftigt. Die Ironie fungiert somit als Mechanismus der Ausgrenzung und der Vergemeinschaftung gleichermaßen. ${ }^{31}$ Schon Schleiermacher hat von der Ironie als einem Hilfsmittel gesprochen ,in der größeren Gesellschaft eine kleinere geheime zu errichten“. Wenn sehr unter-

nen, sind auch die vermeintlich mächtigeren Richter auf die kontinuierliche Kooperation von Zeugen und Angeklagten angewiesen. Zudem ist das allzu rigorose Pochen auf vermeintlichen Machtpositionen ständig durch verfahrenstypische Gegenstrategien (Befangenheitsanträge; bei Staatsanwälten und Verteidigern durch Einsprüche gegen bzw. Zurückweisung von Fragen) bedroht.

${ }^{31}$ Die Grenzen der dabei jeweils zustande kommenden Gemeinschaft sind nicht strikt abzugrenzen. Durch die Ironie wird also nicht nur eine Gemeinschaft der Professionellen erzeugt, wie man vermuten könnte. 
schiedliche Menschen zu einer „Gesellschaft" zusammengezwungen seien

„... so giebt es ja kein höflicheres und freundlicheres Hülfsmittel, als, die Einheit bestehen zu lassen, und zur Herzenserleichterung neben ihr und in ihr, ohne Nachtheil der Anderen, insgeheim, wenn gleich nur auf vorübergehende Augenblicke, eine reizendere und gehaltvolle Vereinigung zu stiften." (Schleiermacher 1878/1984: 183)

Der Aspekt des Humorigen spielt dabei keineswegs die entscheidende Rolle. Allerdings kann er die Vergemeinschaftungswirkung der Ironie positiv unterstützen. ${ }^{32}$

Es bleibt als letztes die Frage zu beantworten, was die besondere „interaktive Leistung" der Ironie für das Gerichtsverfahren und hier speziell im Kontext der Vernehmung von Angeklagten und Zeugen ausmacht. Dazu müssen wir ein wenig ausholen: Nähert man sich dem Phänomen der (Un-)Glaubwürdigkeit im Gerichtsverfahren in einer ethnomethodologisch- konversationsanalytischen Perspektive, dann sieht man sich mit einer eigenartigen Schwierigkeit konfrontiert: Glaubwürdigkeit ist offensichtlich ein sozialer Tatbestand, von dem die Beteiligten in der Situation sehr wohl wissen, um den sie ringen, den sie in Frage stellen usw. - ohne davon ausdrücklich sprechen bzw. ihn feststellen zu müssen. Obwohl anzunehmen ist, daß einigen Zeugen und vielen Angeklagten nicht geglaubt wird, kommt es außerordentlich selten vor, daß Angeklagte und Zeugen bereits während der Beweiserhebung explizit beschuldigt werden, die Unwahrheit zu sagen.$^{33}$ Dies geschieht, wenn überhaupt, erst später in den Plädoyers und in den mündlichen Urteilsbegründungen. Selbst deutliche Bezweiflungen von Zeugenaussagen während der Beweiserhebung sind relativ selten. Aussagen von Angeklagten werden zwar etwas häufiger als unglaubwürdig markiert; in vielen Fällen geschieht dies jedoch ebenfalls nicht. Die beteiligten Juristen, vor allem aber die Richter vermeiden es offenbar, allzu eindeutige

\footnotetext{
${ }^{32}$ Nach Weinrich (1976: 580) gerät die Ironie überhaupt erst im 19. Jahrhundert in eine Begriffsnachbarschaft zum Humor, wobei das Verhältnis beider Begriffe bis heute in einer gewissen Ambivalenz verbleibt.

${ }^{33}$ Manche Juristen führen dies auf fehlerhafte Alltagstheorien oder auch auf schiere Bequemlichkeit der Richter zurück. Üblicherweise versprechen sich diese Autoren Abhilfe durch eine Forcierung des aussagepsychologischen Problembewußtseins (z. B. Reinecke 1986). Wir ziehen es demgegenüber vor, die Richter nicht zu ,aussagepsychologischen Deppen “ zu stilisieren und vermuten interaktionsstrukturelle Gründe für diese - nur vermeintliche - Gutgläubigkeit.
}

Einschätzungen zur Beweislage noch während der Beweiserhebung bekannt zu geben. Gleichwohl besteht hinsichtlich der Würdigung von Zeugen und ihren Aussagen unter den juristischen Verfahrensbeteiligten zumindest in den meisten Fällen eine weitgehende, wenn auch stillschweigende Übereinstimmung. Offensichtlich gelingt es ihnen auch so, d.h. allein in und durch die Art und Weise ihres Fragens und Reagierens für alle praktischen Zwecke Glaubwürdigkeit bzw. Unglaubwürdigkeit in der Situation zu etablieren. Hier scheint uns der funktionale Bezugspunkt der Ironisierung im Verfahren zu liegen. Mit ironischen Äußerungen kann für alle Beteiligten sichtbar ein ,vorläufiges Zwischenergebnis eingefahren" und in der laufenden Verhandlung ein "Meilenstein gesetzt" werden. Unsere Untersuchung hat eine Reihe von Belegen für die These erbracht, daß bei diesem Verständigungsprozeß die kommunikative Modalität der Ironie eine bedeutsame Rolle spielt, und daß die Wirkung der Ironie mit der besonderen Methodizität dieses Äußerungsformates zusammenhängt:

1. Ironisierungen erlauben Bezweiflungen, ohne daß der Zweifel ausdrücklich als solcher markiert und ggf. begründet werden müßte.

2. Mit ihrer Hilfe kann man den Ironieobjekten wie den anderen Anwesenden gegenüber Zweifeln deutlich machen, ohne daß dagegen von den Betroffenen mit großer Aussicht auf Erfolg interveniert werden könnte.

3. Ironisierungen gestatten es, Aufmerksamkeit zu fokusieren und Hinweise zu geben, ohne gleich zu endgültigen Festlegungen greifen zu müssen. Für die ironisierte Person wie für das Publikum wird zwar deutlich markiert, daß eine tendentiell negative Bewertung des Ironieobjekts stattgefunden hat, nicht aber, auf was genau sich diese Beurteilung bezieht und wie sie im Einzelnen ausfällt.

4. Ironisierungen ermöglichen Bezweiflungsphasen abzuschließen und zu neuen Themen überzugehen. In diesem Zusammenhang ist zu bedenken, daß Gerichtsverfahren offensichtlich darauf ausgerichtet sind, es erst ganz am Ende des Verfahrens zu endgültigen Entscheidungen kommen zu lassen. Ein unangenehmer Nebeneffekt dieses verfahrenstypischen „Offenhaltens" ist, daß es keine immanenten Stopregeln für die Befragungs- und Bezweiflungsaktivitäten gibt. Hier kann die Ironie, insoweit es darauf von Seiten der Ironisierten wie des Publikums nichts mehr zu sagen gibt, das Problem einer Kommunikation, die sich im Kreise dreht, lösen helfen. 
5. Mit Ironisierungen lassen sich erhebliche interaktive Wirkungen erzielen, ohne daß die verschiedenen Beteiligten ihr Gesicht verlieren müßten. Das Objekt einer erfolgreichen Ironie ist zwar sozial angeschlagen, nicht aber im Garfinkel'schen Sinn „degradiert“. Auch das Ironiesubjekt und das Publikum geht dabei nicht das Risiko ein, sich auf bestimmte Feststellungen und Absichten festzulegen. Man könnte im Zusammenhang der Verwendung von Ironie im Verfahren von „taktvoller Entschiedenheit" sprechen. ${ }^{34}$

6. Ironisierungen gestatten es schließlich, ein Verfahren in relativ ruhigen Bahnen ablaufen $\mathrm{zu}$ lassen, gleichwohl aber erheblichen Dissens zwischen den Beteiligten zum Ausdruck zu bringen. Sie dienen somit nicht nur der gemeinsamen „Wahrheitsfindung“. Ihre vielleicht noch wesentlichere Leistung besteht paradoxerweise darin, die Ernsthaftigkeit des Verfahrens auf. rechtzuerhalten.

\section{Literatur}

Bergmann, J., 1982: Schweigephasen im Gespräch. S. 143184 in: H.-G. Soeffner $(\mathrm{Hg})$, Beiträge zu einer empirischen Sprachsoziologie. Tübingen: Narr.

Bollnow, O.F., 1958: Die Ironie. S. 147-179 in: Ders., Die Ehrfurcht. Frankfurt/M.: Klostermann.

Brown, P./Levinson, S.C., 1987: Politeness. Some universals in language usage. Cambridge: Cambridge University Press.

Drew, P., 1987: Po-faced receipts of teases. Linguistics 25 : 219-253.

Eco, U., 1984: Nachschrift zum „Namen der Rose“. München, Wien: Hanser.

\footnotetext{
${ }^{34}$ Goffman diskutiert ähnliche Phänomene unter dem Überbegriff der 'andeutenden Kommunikation' (vgl. 1974: $36 \mathrm{f}$.). Gleichwohl steht die ironisierte Person aufgrund des sie immobilisierenden situativen Arrangements ziemlich „dumm“ da. Diese Dummheit bezieht sich in diesem Zusammenhang nicht auf ihre kognitiven Fähigkeiten, sondern ihre soziale Kompetenz. Das Ironieobjekt wird als sozial inkompetent vorgeführt. Dem entspricht, daß die Überprüfung von Aussagen und Behauptungen im Gerichtsverfahren grundsätzlich nach Kriterien sozialer Rationalität erfolgen mu/3. Die „Realität“ der Tat und ihrer Umstände sind im Verfahren nicht mehr zugängig. Unglaubwürdigkeit hat somit nichts mit Wahrheit im Sinne der Abbildgenauigkeit von Aussagen zu tun. sondern ergibt sich sozusagen ,,autopoietisch“ als Resultat von sozialen Konsistenzprüfungen der Aussagen der Beteiligten und den gegebenen praktischen Umständen dieses Verfahrens.
}

Glaser, B.G./Strauss, A.L., 1964: Awareness contexts and social interaction. American Sociological Review: 669679.

Goffman, E., 1974: Das Individuum im öffentlichen Austausch. Frankfurt/M.: Suhrkamp.

Goffman, E., 1980: Rahmenanalyse. Ein Versuch über die Organisation von Alltagserfahrungen. Frankfurt/M.: Suhrkamp.

Günthner, S., 1994: Zwischen Konfrontation und Spiel. Zur kommunikativen Konstruktion von Frotzeleien. Ms. Konstanz/Gießen.

Heritage, J., 1984: Garfinkel and Ethnomethodology. Cambridge: Polity Press.

Japp, U., 1983: Theorie der Ironie. Frankfurt/M.: Klostermann.

Lapp, E., 1992: Linguistik der Ironie. Tübingen: Narr.

Lewandowski, T., 1990: Linguistisches Wörterbuch 2, 5. Auflage. Heidelberg und Wiesbaden: Quelle \& Meyer/ UTB.

Luhmann, N., 1969: Legitimation durch Verfahren. Neuwied und Berlin: Luchterhand.

Luhmann, N., 1980: Interaktion in Oberschichten: Zur Transformation ihrer Semantik im 17. und 18. Jahrhundert. S. 72-161 in: ders.: Gesellschaftsstruktur und Semantik Bd. 1. Frankfurt/M.: Suhrkamp.

Mulkay, M., 1988: On Humor. Its nature and its place in modern society. Cambridge: Polity Press.

Psathas, G., 1995: Conversation analysis. The study of talk-in-interaction. Thousand Oaks: Sage.

Reinecke, G., 1986: Die Krise der Beweiswürdigung im Zivilprozeß oder Über die Schwierigkeit, einem Zeugen nicht zu glauben. Monatsschrift für Deutsches Recht 8: 630-637.

Sacks, H., 1975: An analysis of the course of a joke's telling. S. 337-352 in: R. Bauman/J. Sherzer (eds.), Exploration in the Ethnography of Speaking. Cambridge: Cambridge University Press.

Schleiermacher, F.D.E., 1798/1984: Versuch einer Theorie des geselligen Betragens. In: Kritische Gesamtausgabe, Erste Abteilung: Schriften und Entwürfe, Band 2 (herausgegeben von G. Ebeling u.a.). Berlin/New York: de Gruyter, S. 165-184.

Weinrich, H., 1966: Linguistik der Lüge. Heidelberg

Weinrich, H., 1976: Ironie. S. 578-582 in: Ritter, J./Gründer, K. (Hg.), Historisches Wörterbuch der Philosophie. Bd. 4. Basel: Schwabe.

Wolff, S., 1993: Der Witz und seine soziale Organisation. S. 107-126 in: J. Hohl/G. Reisbeck (Hg.), Individuum, Lebenswelt, Gesellschaft. München: Profil.

Wolff, Stephan, 1994: Glaubwürdigkeit von Zeugen und ihren Aussagen als Handlungs- und Darstellungsproblem. S. 21-36 in: Hof, H. u.a. (Hg.), Recht und Verhalten. Baden-Baden: Nomos.

Wolff, Stephan/Müller, Hermann, 1995: Interaktive Aspekte der Glaubwürdigkeitskonstruktion im Strafverfahren. Kriminologisches Journal 27: 209-226.

Wolff. Stephan et al., 1996: Kompetente Skepsis - Glaubwürdigkeit als Handlungs- und Darstellungsproblem im Strafverfahren. Unveröffentlichter Endbericht an die Volkswagenstiftung. 